\title{
ILCEA
}

Revue de l'Institut des langues et cultures

d'Europe, Amérique, Afrique, Asie et Australie

$12 \mid 2010$

La FASP : dix ans après...

\section{Le substrat culturel spécialisé dans les romans maritimes d'Alexander Kent}

\section{Anthony Saber}

\section{OpenEdition}

\section{Journals}

Édition électronique

URL : http://journals.openedition.org/ilcea/572

DOI : 10.4000/ilcea.572

ISSN : 2101-0609

Éditeur

UGA Éditions/Université Grenoble Alpes

Édition imprimée

ISBN : 978-2-84310-180-9

ISSN : 1639-6073

Référence électronique

Anthony Saber, «Le substrat culturel spécialisé dans les romans maritimes d'Alexander Kent », ILCEA [En ligne], 12 | 2010, mis en ligne le 29 septembre 2010, consulté le 02 mai 2019. URL : http:// journals.openedition.org/ilcea/572 ; DOI : 10.4000/ilcea.572

Ce document a été généré automatiquement le 2 mai 2019.

(c) ILCEA 


\title{
Le substrat culturel spécialisé dans les romans maritimes d'Alexander Kent
}

\author{
Anthony Saber
}

Si l'on adhère au postulat de Petit (1999) selon lequel la fiction à substrat professionnel (FASP) constitue "une autre voie d'accès à l'anglais de spécialité ", on peut formuler l'hypothèse qu'elle constitue un champ d'investigation légitime pour qui souhaite caractériser la culture propre à un milieu, une profession ou une discipline. En effet, si la spécialisation de l'anglais peut être envisagée comme un phénomène mettant en jeu la traditionnelle triade langue-discours-culture, il semble pertinent d'aborder la FASP sous l'angle culturel. À notre connaissance, peu d'études portant sur la FASP ont adopté cette approche, peut-être parce que la définition de la "culture », mot tant galvaudé, parait problématique et évanescente. Mettant en scène Richard Bolitho, officier de la Marine royale britannique au temps de la Révolution française et des guerres napoléoniennes, le cycle de 29 romans d'aventures maritimes publiés par Alexander Kent (alias Douglas Reeman) entre $1968^{1}$ et 2007 nous parait offrir un terrain d'investigation idéal ${ }^{2}$, dans la mesure où, comme l'indiquent Dufoulon et al. (1998) ${ }^{3}$ les milieux maritimes militaires se caractérisent par la densité d'un système de valeurs et de traditions partagées par les membres des équipages.

1 Nous tenterons donc de montrer que, même si le cycle romanesque des aventures de Richard Bolitho ne correspond pas stricto sensu aux critères définitoires de la FASP, il recèle un substrat spécialisé étendu, dont la composante culturelle nous semble prédominante.

\section{Le roman d'aventures maritimes, aux marges de la FASP}

Avec Herman Melville (White Jacket, Moby Dick), Richard Henry Dana (Two Years before the Mast), Joseph Conrad, ou, plus récemment, Nicholas Monsarrat (The Cruel Sea) et Herman 
Wouk (The Caine Mutiny), la littérature anglo-américaine compte de nombreux auteurs de fictions ayant trait à l'univers maritime ${ }^{4}$. Deux représentants plus récents du genre, C. S. Forester et Patrick O'Brian, ont adopté une approche sans doute plus formulaïque du sea yarn: chez ces deux auteurs, l'œuvre se déploie en un vaste cycle de romans ${ }^{5}$ mettant en scène des héros récurrents (Horatio Hornblower chez le premier, l'officier Jack Aubrey et le médecin Stephen Maturin chez le second). Chez les deux auteurs, le cadrage chronologique est quasi-identique (l'action des romans se situe en marge d'évènements historiques au temps de la Révolution française et des guerres napoléoniennes); de même, Hornblower et Aubrey sont calqués sur un personnage réel, le contre-amiral Thomas Cochrane, marin célèbre pour ses succès contre les flottes française et espagnole ${ }^{6}$

Les romans maritimes d'Alexander Kent adoptent les mêmes principes narratifs. Son héros fétiche, Richard Bolitho, commence sa carrière comme jeune commandant du brick Phalarope en 1793 (période couverte par l'ouvrage To Glory We Steer); amiral, il meurt au combat en 1814 (comme nous l'apprenons dans Sword of Honour) après avoir parcouru toutes les mers du globe et participé à de très nombreuses batailles, dont celles d'Aboukir et de Trafalgar. Chez Kent, tout comme chez Forester et O'Brian, la narration est conforme aux caractéristiques que Tadié (1996) assigne au roman d'aventures; en premier lieu, le « suspens » constitue le ressort principal de l'intrigue :

Le roman d'aventures organise son suspens de telle sorte qu'aucun événement ne porte en lui-même de signification immédiate, que la solution (en termes de vie ou de mort) comme l'explication (en termes de vérité ou d'erreur) en soient toujours différées. [...] La force du suspens qui, en soi, n'est pas esthétique, est de supporter tous les contenus que son flot irrésistible charrie avec lui : c'est ainsi que Jules Verne fait absorber les descriptions. (Ibid., p. 8)

En outre, le héros de l'intrigue est protégé de tout événement tragique :

La conviction qu'il ne peut rien arriver de catastrophique, de mortel au héros principal, aux protagonistes sympathiques, fait partie, par-delà la peur, des règles du genre [...] le roman d'aventures rejoint ici le conte de fées, la littérature enfantine, le roman sentimental. (Ibid., p. 10)

Enfin, le troisième pilier de l'esthétique du roman d'aventures est le déplacement dans l'espace, déplacement soumis à un délai impérieux :

Depuis les Grecs, il n'y a pas de roman d'aventures sans course ni fuite ; la rapidité du déplacement triomphe du temps: les personnages sont toujours menacés d'arriver trop tard. (Ibid., p. 36)

6 Ces trois caractéristiques majeures sont patentes dans tous les romans maritimes de Kent. Ainsi, dans To Glory We Steer, Bolitho reçoit en 1782 le commandement du brick Phalarope et, après avoir triomphé d'un début de mutinerie de l'équipage, traverse l'Atlantique pour combattre aux Antilles la flotte de l'amiral français De Grasse et ses alliés américains. obligé de livrer bataille à son propre frère, qui a rejoint les rangs des insurgés américains et commande l'Andiron, navire de la jeune marine américaine, il traverse de nombreuses péripéties et finit par prendre part à la bataille des Saintes ${ }^{7}$ (9-12 avril 1782), à laquelle il survit, alors que son équipage est décimé par le feu ennemi.

7 Ainsi, on voit que les romans maritimes de Kent ne répondent pas stricto sensu aux critères canoniques de la FASP décrits par Petit (1999). Si ces ouvrages s'inscrivent incontestablement dans la veine du roman commercial et prennent pour objet un milieu professionnel particulier, celui des marins militaires, ils ne font pas du substrat professionnel un des principaux ressorts narratifs de l'intrigue. Aux marges de la FASP, 
les romans de Kent contiennent cependant de nombreux éléments constitutifs d'un substrat spécialisé que nous nous proposons de détailler. Mais, puisque nous nous préoccupons ici de la composante culturelle du substrat spécialisé, il convient tout d'abord de préciser ce que nous entendons par « culture».

\section{La dimension culturelle du substrat spécialisé}

8 Dans le champ des sciences humaines, le mot «culture " a reçu tant d'acceptions différentes qu'il semble impossible d'en dresser ici une liste exhaustive ${ }^{8}$. Williams (1983, p. 87) n'hésite d'ailleurs pas à affirmer: "Culture is one of the two or three most complicated words of the English language ». Tentant un historique de cette notion, Baldwin, Faulkner et Hecht (2006) déplorent sa nature fondamentalement évanescente. Explorant les définitions de la culture proposées par divers auteurs depuis le XVIII ${ }^{\mathrm{e}}$ siècle, Kroeber et Kluckhohn (1952) identifient plus de 150 acceptions différentes relevant de champs disciplinaires multiples: philosophie, sociologie, psychologie et ethnologie. Ils proposent une typologie de ces définitions en identifiant 6 groupes distincts: les définitions énumératives ${ }^{9}$ décrivent la culture comme une liste de contenus; les définitions historiques ${ }^{10}$ mettent l'accent sur la tradition et les processus de transmission; les définitions normatives interprètent la culture comme un code plus ou moins rigide d'idéaux et de comportements attendus; les définitions psychologiques prennent pour principal objet les comportements d'apprentissage et de résolution des problèmes; les définitions structuralistes tentent d'identifier des schémas récurrents dans l'économie interne d'une culture; et enfin les définitions " génétiques ${ }^{11}$ insistent sur les symboles, les idées et les artefacts. Cette nomenclature les conduit à proposer une définition synthétique, qui paraît somme toute recevable ${ }^{12}$ :

Culture consists of patterns, explicit and implicit, of and for behavior acquired and transmitted by symbols, constituting the distinctive achievements of human groups, including their embodiments in artifacts; the essential core of culture consists of traditional (i.e., historically derived and selected) ideas and especially their attached values; culture systems may, on the one hand, be considered as products of action, on the other as conditioning elements of further action. (Ibid., p. 181)

Dans un ouvrage plus récent, Baldwin et al. (2006) recensent au total près de 300 définitions du mot «culture». Le nombre de définitions s'est donc considérablement accru dans la deuxième moitié $\mathrm{du} \mathrm{xx}^{\mathrm{e}}$ siècle. Parmi les définitions les plus citées, on notera celle de Clifford Geertz (1973, p. 4-5), qui envisage la culture comme un ensemble de réseaux sémiotiques :

The concept of culture I espouse [...] is essentially a semiotic one. Believing, with Max Weber, that man is an animal suspended in webs of significance he himself has spun, I take culture to be those webs, and the analysis of it to be therefore not an experimental science in search of law but an interpretative one in search of meaning.

Si le nombre de définitions du mot « culture » parait donc très élevé, peu d'analystes ont parlé de « cultures spécialisées ». Challe (1988, p. 49) identifie par exemple des «cultures d'experts » comme un ensemble de pratiques spécialisées transmises par l'éducation et la formation, ces pratiques pouvant relever du champ social, religieux, culturel ou politique. Prenant acte de la polysémie du mot «culture», Gotti (2008, p. 221) propose une définition de la culture dans le contexte d'une discipline juridique (l'arbitrage) : « $a$ complex set of traditions and standardised social practices peculiar to a specific professional group $»$. 
Ce bref tour d'horizon semble démontrer que :

- il semble illusoire de vouloir définir de manière définitive le mot « culture »;

- quelle que soit la définition adoptée, la matérialité objective de la culture ne paraît pas contestable : à tout le moins, elle constitue un facteur de viscosité qui gouverne partiellement les comportements et les représentations d'un groupe humain, dont elle trace en quelque sorte les contours ;

- regroupant des éléments de nature hétérogène, la culture se caractérise par son caractère composite ;

- en renversant le principe du rasoir d'Occam (pluralitas non est ponenda sine necessitate), censé fonder toute démarche scientifique, on peut se demander si la diversité des définitions disponibles ne correspond pas à une nécessité, c'est-à-dire si, en définitive, toute définition de la culture ne peut être qu'adaptative, modulable en fonction de l'objet observé par l'analyste.

Nous adopterons donc une démarche empirique en recherchant dans le substrat spécialisé présent dans les romans d'Alexander Kent ce qui pourrait relever du culturel, et en adoptant pour ce faire une définition à la fois très extensive et a minima de la culture, que nous pourrions envisager comme une configuration stabilisée d'éléments hétérogènes propre à un groupe humain et constituée en patrimoine commun. On peut établir une première distinction à ce point entre ce substrat culturel dont nous supposons l'existence, et les deux composantes déjà bien identifiées du substrat spécialisé présent dans la FASP. Le substrat culturel s'ajouterait en effet au substrat disciplinaire (comme l'indique Petit dans son article séminal en 1999, la FASP a ceci de particulier qu'elle s'attache à décrire de manière précise les savoirs et les procédures des professionnels qu'elle met en scène), et au substrat langagier (la FASP constitue en effet une voie d'accès aux pratiques langagières d'acteurs sociaux spécialisés tels que les juges, les médecins, les militaires, etc.). Ce substrat spécialisé semble bien présent dans les ouvrages de Kent, comme nous allons le démontrer à présent.

\section{Le substrat spécialisé dans les romans maritimes d'Alexander Kent}

\section{Le substrat langagier}

13 Comme d'autres fictions prenant pour cadre un milieu spécialisé, les romans maritimes de Kent contiennent un certain nombre de termes spécialisés. En l'espèce, ces termes ont trait à la description des bâtiments de guerre, de leur gréement, des manœuvres, et de la vie à bord. Considérons ce passage de To Glory We Steer (roman que nous désignerons désormais par le sigle TGWS) :

Richard Bolitho climbed the ladder to the quarterdeck and made himself walk slowly to the weather rail. The frigate was alive with fresh activity, and he could see men standing at the capstan bars, while others waited below the masts with their petty officers. He gauged the wind against his cheek and glanced quickly aloft at the masthead pennant. The ship tugged at her cable eagerly and fretfully [...]. (TGWS, p. 29)

À l'évidence, l'emploi par Kent de termes tels que ladder (l'échelle), quaterdeck (la dunette), weather rail (le bastingage au vent), capstan bars (les barreaux du cabestan), petty officers (sous-officiers), masthead pennant (la flamme en tête de mat) et cable (la chaîne de l'ancre) traduit son souhait de viser un lectorat connaisseur des choses de la mer, même 
si la terminologie employée ne paraît pas hautement spécialisée. Pour autant, le substrat langagier présent dans les textes de Kent ne semble pas très développé, pour la raison évidente que, à l'inverse de la plupart des auteurs de FASP (souvent anciens praticiens du milieu qu'ils décrivent, à l'image de John Grisham, ancien avocat), il n'avait pas accès, en rédigeant ses romans, à la manière dont s'exprimaient les équipages britanniques au temps de la marine à voile. Dans quelques rares passages, Kent, lui-même ex-officier de la Royal $\mathrm{Navy}^{13}$, s'essaie néanmoins à restituer la phraséologie maniée par certains membres de l'équipage. Par exemple, dans ce passage de Form Line of Battle! (désormais FLB), l'homme d'équipage chargé d'effectuer les relevés de sonde à l'avant du navire (le leadsman) annonce ainsi une profondeur de 20 brasses $^{14}$ :

[...] From forward came a splash followed by the leadsman's droning chant, "By th'mark twenty!" (FLB, p. 115)

Cependant, bien que Kent, en tant qu'ancien marin, connaisse le jackspeak, ce jargon propre aux marins de la Royal Navy' ${ }^{15}$, on n'en trouve pas trace dans ses romans. Sans doute n'a-t-il pas voulu se risquer à tenter de reconstituer une version artificiellement vieillie de ce jargon propre au Jack Tar, l'archétype du matelot britannique.

\section{Le substrat disciplinaire}

Le substrat disciplinaire paraît plus présent dans les romans de Kent que le substrat langagier. Plusieurs disciplines sont en effet évoquées par Kent avec un certain souci de précision documentaire : la manœuvre, bien entendu, et, de manière sans doute plus allusive, les transmissions, l'artillerie, et la médecine de guerre. Comme les romans de Kent se déroulent au temps de la marine à voile, la manœuvre est bien évidemment au premier plan. Le récit est en effet constamment rythmé par les manœuvres qu'exécute l'équipage, sous les ordres de Bolitho. La manœuvre comprend deux sous-disciplines : la manœuvre du bâtiment (c'est-à-dire le contrôle de ses évolutions sur le plan d'eau), et la manipulation du gréement. Les évènements obligent ainsi fréquemment Bolitho à ordonner des changements de cap; dans le passage ci-dessous, il donne l'ordre à son maitre d'équipage Quarme de se porter à la rencontre de six vaisseaux de ligne :

Quarme said formally, "Masthead reports six sail of the line to the north, sir." [...]

"Very well. Alter course two points to larboard to intercept." (FLB, p. 30-31)

17 S'adressant principalement à un lectorat de connaisseurs de la chose maritime, Kent n'hésite pas à évoquer les manipulations du gréement de manière très technique, comme dans ce passage où il ordonne à son équipage de carguer les perroquets et les bassesvoiles pour ne garder que les huniers et les focs :

Bolitho said suddenly "Hands aloft, Mr. Quarme. Get the topgallants and courses in, if you please. Tops's and jib will suffice for our purposes." (FLB, p. 42) Véritable ressort de l'intrigue, la manœuvre constitue donc chez Kent l'essentiel du substrat disciplinaire, même si d'autres disciplines sont décrites, sans doute plus schématiquement. Dans ce passage où Bolitho, commandant le vaisseau Hyperion, est informé par la vigie d'un message provenant du navire Snipe, Kent évoque ainsi le système de transmission de signaux par pavillons inventé en 1762 par l'amiral britannique Richard Howe ${ }^{16}$ :

"Deck there! Signal reads, "Snipe to Hyperion. Strange sail bearing nor'-nor'west." [...] Bolitho said [...] "Very well. Make to Snipe. [...] "Investigate immediately" [...] Then signal the convoy to reduce sail." (FLB, p. 159). 
L'artillerie n'est pas négligée. Ainsi, lorsque Bolitho s'apprête à utiliser ses caronades contre un navire français, Kent nous explique les particularités de cette pièce d'artillerie :

[Bolitho] stared down at the nearest weapon, snub-nosed and ugly, and lacking either the grace or the proportion of a proper deck gun. Yet a carronade could throw a massive sixtyeight-pound shot at short range, the power of which was devastating. Each circular shot burst on impact to deluge everything nearby with murderous cast-iron balls. One shot had the lethal quality of grape, added to which was the weight of a much heavier weapon. (TGWS ,p. 353-354)

Cependant, ces passages à dimension didactique, qui constituent comme l'on sait une des particularités de la FASP, demeurent relativement rares chez Kent, qui ne met pas en avant le substrat disciplinaire ${ }^{17}$. C'est donc, à notre sens, le substrat culturel qui est mobilisé à titre principal par Kent pour donner chair au monde maritime qu'il évoque.

\section{Le substrat culturel spécialisé}

En nous appuyant sur la définition du mot « culture » que nous avons proposée plus haut, et en prenant acte de son caractère hétérogène et composite, il apparaît que le matériau culturel est constitué en substrat culturel spécialisé dans les romans maritimes de Kent. Celui-ci comprend à notre sens trois éléments: les traditions des gens de mer, le cérémonial, et le jus in bello (c'est-à-dire les règles coutumières, implicitement acceptées par tous, encadrant les actions militaires).

\section{Les traditions des gens de mer}

22 Il semble que les gens de mer partagent un corpus de traditions solidement établies, résultat d'une cristallisation culturelle s'étalant sur plusieurs siècles. Bronner (2007) montre, par exemple, l'universalité des rites initiatiques organisés à l'occasion du passage de l'équateur par un navire ${ }^{18}$. En s'appuyant sur des sources littéraires et des récits de marins ${ }^{19}$, Rediker (1989, p. 153-186) fait l'inventaire de ce corpus hétéroclite de traditions, de croyances et de superstitions. Selon lui, ce corpus culturel se développe dans le cadre d'une socialisation de substitution, alors même que l'équipage est confronté au défi de construire, au milieu d'un environnement hostile, une société autonome :

First and foremost was the simple but profound fact of the ship's isolation, its social and geographical distance from landed society and the church. The deinstitutionalized setting of seafaring life allowed maritime culture to develop in a relatively autonomous way, since seamen lived apart from the conventions, symbols and rituals of mainstream religious society for extended periods. (Ibid., p. 173-174)

Agrégeant des éléments de nature hétérogène, ce corpus culturel frappe par son aspect syncrétique :

The seaman's worldview combined Christian and pre-Christian beliefs, referents, and orientations. Seamen used classical mythology; biblical tales, and traditional yarns as they created their lore and their pantheon of meaningful figures. [...] Charon and the River Styx appeared before dying seamen; Neptune figured with increased prominence in seafaring rituals. (Ibid., p. 184-185)

Rediker mentionne également des superstitions universellement répandues chez les gens de mer, comme le fait de placer des pièces de monnaie sous le mât, afin de protéger l'équipage des mauvais esprits et de lui garantir un retour sans encombre au port d'attache. Volo \& Volo (2002, p. 156) signalent par ailleurs que, au temps de la marine à 
voile, les équipages voyaient dans la présence de femmes à bord du navire une source de malheurs ${ }^{20}$.

Certaines traditions observées chez les gens de mer ont trait au commandant du navire. Rediker (1989, p. 154) indique ainsi que sa solitude est totale, à la fois parce que l'équipage n'attend pas de familiarité de sa part, et parce qu'il utilise la distance pour imposer son autorité. La tradition impose que cette autorité soit absolue, et Kent ne manque pas de noter qu'elle va au-delà de l'autorité officielle qui lui est conférée par une autorité officielle extérieure (l'Amirauté britannique pour Bolitho, en l'occurrence) :

Every man aboard would know that the straight-backed figure in the gold-laced hat was [...] the man who, next to God, held complete sway over every life in his ship. One who could flog or hang, just as he could equally reward and recognise the faults or efforts of everybody under his hand. (Ibid., p. 9)

\section{Le cérémonial}

Kent évoque à plusieurs reprises le cérémonial qui accompagne certains évènements de la vie à bord. La principale fonction de ce cérémonial semble résider dans la matérialisation de la structure hiérarchique qui gouverne la micro-société du navire. Chez Kent, le cérémonial sert avant tout à marquer le respect envers l'autorité. Ainsi, alors que son navire se porte à la rencontre d'un vaisseau de ligne britannique à bord duquel se trouve un amiral, Bolitho ordonne que l'on tire une salve pour marquer son respect envers cet officier plus gradé que lui (le nombre important de pièces mises en action dénote l'importance de ce geste symbolique) : «Stand by to fire salute! Eleven guns, if you please! » ( TGWS, p. 115). Ce rapport du cérémonial à la structure hiérarchique de la société militaire est également visible lorsque Bolitho affirme son autorité en lisant à voix haute, devant tout l'équipage, la lettre le reconnaissant commandant du navire :

He read his commission briskly and without emotion. It was addressed to Richard Bolitho, Esquire, from Samuel Hood, Admiral of the Red, and required him to take upon him the charge and command of captain in his Britannic Majesty's ship Hyperion. Most of the men had heard such commissions read before, some no doubt many times, yet as he read through the neat, formal phrases he was conscious of the silence. As if the whole ship were holding her breath. (Ibid., p. 11)

On constate que ce cérémonial semble bien établi. La lecture de cette lettre n'est pas formellement imposée par l'Amirauté, mais tout commandant doit se conformer à cette coutume, que lui impose la présence vive d'une historicité partagée. Comme le note Rivière (1988, p.141-142), «la cérémonie collective a les effets d'une tradition en ce qu'elle répète, dans un style rigide, des formes et contenus de représentations que les participants ont en commun ».

28 La nature culturelle du cérémonial ne fait guère de doute : sa valeur principale réside moins, en effet, dans son scénario concret que dans sa portée symbolique. Décrivant une cérémonie propre aux militaires, la parade, Alexis Philonenko (1974, p. 38-39) y voit un «phénomène de langage »:

La parade est, dans la mesure où elle est « monstration », déploiement symbolique.

[...] Les drapeaux et les oriflammes, la tenue des hommes arborant leurs décorations, la disposition des troupes [...] sont autant de moments symboliques qui rehaussent les éléments destinés à être une pure « monstration » de la force.

Kent sait faire sentir au lecteur la portée symbolique du cérémonial observé chez les marins. Décrivant par exemple une cérémonie encore en vigueur dans toutes les marines de guerre du monde (l'accueil d'une autorité à la coupée du bâtiment), Kent traduit les 
craintes de Bolitho, qui, se préparant à accueillir à bord de son navire l'amiral Napier, jette un dernier regard inquiet sur le pont supérieur du navire, sachant que l'amiral se montrera impitoyable envers lui lorsqu'il inspectera son bâtiment. La cérémonie de l'accueil à la coupée symbolise ainsi le transfert, certes temporaire, mais néanmoins total, de son autorité à une autorité extérieure :

Rear-Admiral Sir Robert Napier stepped through the Phalarope's entry port and removed his hat to receive his due respects. As the shrill pipes faded into silence and the marine guard presented arms, the frigate's small drummer, accompanied by two reedy fifes, broke into a frail but jaunty march, and with a final glance around the upperdeck Bolitho stepped forward to meet his admiral. (TGWS, p. 251)

La culture propre aux marins se déploie donc de manière ostensive dans le cérémonial. Mais elle peut aussi se manifester de manière plus discrète et plus implicite, notamment en matière de règles s'appliquant aux confrontations armées : le jus in bello.

\section{Le jus in bello}

31

Kent met fréquemment en scène dans ses ouvrages des confrontations armées entre l'équipage que commande Bolitho et les adversaires de l'Angleterre (principalement des marins français). On découvre à cette occasion que, même si les combats sont acharnés, la guerre totale n'est pas de mise entre gens de mer. Ainsi, lorsque tous les moyens de résister ont été épuisés, il n'est pas déshonorant de se rendre à un adversaire qui a combattu dans l'honneur. Dans ce cas, le commandant du navire défait signale sa reddition en amenant le pavillon national qui flotte à la poupe du bâtiment : c'est un code compris de tous les gens de mer. De par sa force coutumière, le jus in bello impose alors de cesser le combat. On voit ainsi dans le passage ci-dessous que le commandant français du navire que Bolitho vient de vaincre en le désemparant ne souhaite pas encore déclarer forfait :

The Tricolour still flapped from the gaff, and the musketry was a clear answer to [Bolitho's] plea to end this one-sided fight. (Enemy in Sight, désormais EIS, p. 70)

\section{Vers une caractérisation des cultures spécialisées}

Les romans d'aventures maritimes de Kent dressent donc un "portrait culturel » des équipages britanniques au temps de la marine à voile, fût-ce sous une forme stylisée. Ceci permet d'esquisser quelques éléments de définition des cultures spécialisées, c'est-à-dire des cultures propres à un milieu, une profession ou une discipline.

\section{La fonction cohésive de la culture spécialisée}

Chez Kent, une fonction essentielle du capital culturel commun à l'équipage ressortit à la cohésion sociale à bord du bâtiment. Cette cohésion est en effet potentiellement menacée par deux dangers: le risque de confrontation entre les différentes populations spécialisées à bord du navire de guerre, véritable société alvéolaire qui fait cohabiter les fusiliers, les manœuvriers, les artilleurs, les gabiers, etc.; et le risque permanent de mutinerie, d'autant plus présent que la majorité des hommes d'équipage sont issus de la "presse », ce système de recrutement forcé que la Marine britannique utilisait pour gréer ses navires en hommes : 
After weeks of fretting and fruitless interviews [Bolitho] had been given the task of recruiting unwilling inhabitants of the Medway towns to fill the ships which were at last being called into commission. (FLB, p. 6-7) plusieurs appartenances concentriques. De fait, les équipages de Bolitho apparaissent comme dépositaires de cultures multiples : celle des gens de mer au sens large, celle de la Royal Navy, celle du bâtiment sur lequel ils sont affectés, et celle des différents sousgroupes s'agrégeant à bord, de manière plus ou moins informelle. Comme l'ont montré certaines études de sociologie militaire ${ }^{21}$, ce sont probablement les cultures locales (celle du bâtiment, mais surtout celle du "groupe primaire ») qui sont les plus prégnantes, au détriment des macro-cultures extérieures au bâtiment :

Sometimes [these men] were starved by miserly captains, flogged by tyrants, or treated like animals by others. Yet when the call came they rarely failed. It was something which Bolitho never really quite understood. Some said it was out of fear, others that the inbuilt tradition and harsh discipline of the Navy were the real reasons. But he believed it went far deeper. A man-o-war ${ }^{22}$ was a way of life. The Cause and the Flag often came second to the love of the men around her crowded decks. They fought to protect each other, to avenge old comrades lost in forgotten battles. And they fought for their ship. (FLB, p. 278-279) « cellulaire » de la société militaire, du moins chez les hommes du rang. La solidarité des hommes au combat est avant tout une solidarité de proximité ; face au danger, on ne fait plus allégeance au drapeau, mais au premier cercle de ses camarades ; à cela se superpose une volonté de ne pas trahir les camarades déjà tombés au combat, c'est-à-dire la présence d'une historicité implicite.

Le sentiment d'appartenance repose donc sur un processus de segmentation et de différenciation. Celui-ci peut être interne au navire, et se réaliser dans les microsolidarités que nous venons de décrire. Mais la culture des équipages fonde également la «signature » d'un bâtiment particulier, cette «manière de faire " qui le différencie 
subtilement des autres bâtiments de la Royal Navy. Nous pouvons parler ici d'un style collectif différenciateur, au sens où l'entendent Deal \& Kennedy (1982, p. 24) : « For those who hold them, shared values define the fundamental character of their organization, the attitude that distinguishes it from all others ». Cette « signature» se traduit notamment, chez Kent, par une certaine manière d'exécuter les manœuvres, une façon particulière d'exceller dans le maniement du gréement :

Her sail drill and manoeuvring were impeccable, and behind him Bolitho heard Gossett murmur, "Cap'n Winstanley has the feel of the old lady well enough." From him that was praise of the highest order. (EIS, p. 28)

Observant depuis le navire commandé par Bolitho un autre bâtiment britannique commandé par Winstanley, le maître d'équipage Gossett ne peut retenir son admiration devant la virtuosité technique du capitaine Winstanley. Ceci signale sans doute, de manière allusive, l'existence d'une quatrième composante de la culture des équipages, à savoir la maîtrise de certaines habiletés permettant d'apposer une marque unique sur des gestes techniques pourtant communs à toutes les escadres de la Royal Navy. Mais il s'agit là, probablement, d'une sorte d'ineffable culturel, inaccessible à l'analyste dès lors que l'accès au milieu spécialisé repose sur une représentation fictionnelle.

\section{Conclusion}

En définitive, la FASP apparaît comme une voie d'accès légitime à la culture d'un milieu professionnel spécialisé (le milieu des marins militaires en l'occurrence), même si cette culture, agrégation d'éléments hétérogènes constitués en capital commun par une communauté humaine spécialisée, demeure difficile à définir. Le substrat culturel qui se manifeste dans certaines FASP peut donc, au même titre que le substrat langagier ou le substrat disciplinaire, constituer un objet d'étude en anglais de spécialité, à condition de tenter de comprendre ce que recouvre le mot « culture » chez les acteurs spécialisés que l'on se propose de caractériser.

41 S'agissant des marins militaires, il ressort que l'acculturation au milieu professionnel s'appuie avant tout sur une mémoire collective soigneusement entretenue par l'institution militaire. On note ainsi que les grandes marines militaires modernes intègrent une forte dimension culturelle à la formation initiale de leurs personnels. Mack, Seymour et McComas (1998), auteurs d'un guide destiné à l'édification des officiers de l'US Navy, n'hésitent pas à consacrer un chapitre entier (ibid., p. 89-103) aux «traditions et coutumes de la Marine ", c'est-à-dire essentiellement aux règles d'étiquette et de commensalité à bord du navire. De même, Connell \& Mack $(2004$, p. 3) signalent dès l'entame d'un ouvrage comparable que le corpus culturel de l'US Navy sert principalement à préserver la cohésion et l'esprit de corps au sein des personnels :

[...] traditions, ceremonies, [and] customs [...] exert a profound influence upon human behavior. The effect is particularly marked in such professions as the military. Organizations that impose discipline lend themselves to passing on and perpetuating venerated customs, heroic traditions, and dignified ceremonies. Such stimuli, when appreciated and properly applied, inculcate ideals and esprit de corps of incalculable value.

La prégnance d'une culture commune au statut quasi-institutionnel est un trait commun à toutes les grandes marines militaires. On remarque par exemple que, à l'instar de leurs homologues américains, les officiers de marine français sont tenus de connaître les 
principes de comportement décrits dans le fameux «Vercken $»^{23}$, un véritable manuel de maintien intitulé Marine et bons usages.

À l'inverse de la très grande majorité des auteurs de FASP, qui dépeignent des milieux spécialisés contemporains qu'ils connaissent à merveille pour les avoir longuement fréquentés, Alexander Kent ne peut bien entendu nous livrer qu'une version reconstituée, et donc sans doute stylisée, de la culture propre aux marins britanniques au temps de la marine à voile. Mais en raison de la permanence et du caractère patrimonial de cette culture maritime particulière, on peut formuler l'hypothèse que le substrat culturel spécialisé qui se manifeste dans ses romans n'est pas purement fictionnel, mais revêt au contraire une forte authenticité. Familier de la Royal Navy des années 1940 et 1950, c'est en héritier de cette culture groupale transmise d'équipage en équipage qu'Alexander Kent nous donne accès aux représentations et aux valeurs d'un milieu spécialisé aujourd'hui disparu.

\section{BIBLIOGRAPHIE}

\section{Romans d'Alexander KENT}

Enemy in Sight!, [1970], Londres, Random House, coll. « Arrow », 2002.

Form Line of Battle!, [1969], Londres, Random House, coll. « Arrow », 2002.

To Glory we Steer, [1968], Londres, Random Century Group, coll. « Arrow », 1990.

\section{Références}

ARNOLD, M. [1869], Culture and Anarchy, Charleston, BiblioBazaar, 2007.

BALDWIN, J. R., FAULKNER, S. L., HECHT, M. L., " A moving target: The illusive definition of culture », dans Baldwin et al., 2006, p. 3-26.

BALDWIN, J. R., FAULKNER S. L., HECHT, M. L. \& LINDSLEY, S. L. (dir.), Redefining culture: Perspectives across the disciplines, Mahwah, Lawrence Erlbaum Associates, 2006.

BENEDICT, R. [1931], The Science of Custom in the Making of Man, New York, Morrow, citée dans Kroeber \& Kluckhohn, 1952, p. 81.

BOAS, F. [1898], « Publications of the Jesup North Pacific Expedition », dans Stocking G. W., A Franz Boas Reader, Chicago, University of Chicago Press, 1989, p. 107-119.

BODLEY, J. H., Cultural anthropology, Mountain View, Mayfield, 1997.

BRONNER, S. J., Crossing the line. Violence, Play, and Drama in Naval Equator Traditions, Amsterdam University Press, 2007.

CHALLE, O., « Les cultures en discours, trame de fond du français de spécialités », dans BERTRAND, O., SCHAFFNER, I. (dir.), Le français de spécialité. Enjeux culturels et linguistiques, Palaiseau, Éditions de l'Ecole Polytechnique, p. 49-67, 2008.

COATES C., Pellegrin R., Military Sociology, University Park, The Social Science Press, 1965. 
CONNELL R. W., MACK W. P., Naval Ceremonies, Customs, and Traditions, Annapolis, Naval Institute Press, 2004.

CUCHE D., La Notion de culture dans les sciences sociales, Paris, Éditions La Découverte, 2001.

DEAL T., KENNEDY A., Corporate Cultures, Reading, Addison-Wesley, 1982.

DUfoulon S., TROMPETTE P., SAGLIO J., Les Gars de la Marine. Ethnographie d'un navire de guerre, Paris, Métaillié. 1998.

GEERTZ C. [1973], The Interpretation of cultures, New York, Basic Books, 2003.

GOTTI M., «Cultural constraints on arbitration discourse », dans BHATIA, V., CANDLIN, C., Legal Discourse Across Cultures and Systems,Hong Kong University Press, 2008.

KRoeber A. L., KLUCKHoHn C., Culture: A Critical Review of Concepts and Definitions, Harvard

University, Cambridge, Papers of the Peabody Museum of Archaeology and Ethnology, vol. XLVII, $n^{\circ} 1,1952$.

JOLLY R., Jackspeak, Liskeard, Maritime Books, 2000.

MACK W. P., SEYMOUR H. A., MCCOMAS L. A. [1943], The Naval Officer's Guide, Annapolis, Naval Institute Press, 1998.

PETIT M., " La fiction à substrat professionnel : une autre voie d'accès à l'anglais de spécialité ", ASp, n² 23/26, p. 57-81, Bordeaux, GERAS, 1999.

PHILONENKO A., « Guerre et langage », Études polémologiques, n 14, 1974, p. 35-55,

REDIKER M., Between the Devil and the Deep Blue Sea, Cambridge, Cambridge University Press, 1989.

RIVIÈRE C., Les Liturgies politiques, Paris, PUF, 1988.

SANTRAUD J.-M., La mer et le roman américain dans la première moitié du dix-neuvième siècle, Paris, Didier, 1972.

STERLING C. H. , Military communications from Ancient Times to the $21^{\text {st }}$ Century, Santa Barbara, ABCCLIO, 2007.

STOUfFER S. et al. (dir.), The American Soldier, Princeton, Princeton University Press, 1949.

TADIÉ J.-Y. [1982], Le Roman d'aventures, Paris, Presses Universitaires de France, coll. « Quadrige », 1996.

тномаs D. S. , Cochrane: Britannia's Sea Wolf, Annapolis, Naval Institute Press, 2002.

TYLOR E. B. [1871], Primitive culture: Researches into the development of mythology, philosophy, religion, art and custom, 2 vol., Londres, Murray, 1920. [En ligne] http://www.archive.org/bookreader VERCKEN R., Marine et bons usages, Brest, Éditions de la Cité, 1986.

volo J. M., volo D., Daily Life in the Age of Sail, Westport, Greenwood Publishing Group, 2002. WILLIAMS R. [1976], Keywords. A Vocabulary of Culture and Society, New York, Oxford University Press, 1983.

\section{NOTES}

1. Date de publication de To Glory We Steer, premier ouvrage du cycle. 
2. La présente étude prend appui sur un échantillon de trois romans d'Alexander Kent : To Glory We Steer, Enemy in Sight, et Form Line of Battle! (voir bibliographie). Les exemples que nous citons proviennent exclusivement de ces trois ouvrages. Notons que la quasi-totalité des romans maritimes d'Alexander Kent ont été traduits en français dans la collection "Phébus Libretto " (Éditions Phébus, Paris) ; mais nous avons préféré utiliser, dans la présente étude, des citations tirées des ouvrages en anglais.

3. Menant un travail ethnographique à bord de la frégate française Georges Leygues, ces sociologues mettent en effet en évidence une identité sociale propre à ce bâtiment. Cette identité est d'aspect composite et comprend notamment des éléments mémoriels, des rites de passage, un certain esprit de corps, des règles de commensalité, et un grand nombre de traditions. Elle à la fois invisible et présente, manifestant un « sens commun » du bord, et se constitue en mécanisme de régulation sociale pour préserver la stabilité $\mathrm{du}$ « nous » de l'équipage.

4. On trouve chez Santraud (1972) une cartographie du genre pour le domaine américain.

5. La série des Hornblower compte 11 romans publiés entre 1937 et 1967 ; quant au cycle des Aubrey/Maturin de Patrick O’Brian, il compte 20 romans publiés entre 1969 et 1999.

6. Le 6 mai 1801, il s'illustre notamment en capturant la frégate espagnole El Gamo, dont l'équipage dépasse 300 hommes, alors qu'il ne commande qu'un sloop (un brick, c'est-à-dire une frégate de petit tonnage), le Speedy, sur lequel il ne dispose que d'une cinquantaine d'hommes. Son existence haute en couleur est notamment narrée dans une biographie récente (Thomas, 2002).

7. Iles situées entre la Guadeloupe et la Dominique, dans les Antilles.

8. Selon Williams (1983, p. 87-91) et Cuche (2004), le mot «culture » commence à prendre un sens métaphorique au $\mathrm{XIX}^{\mathrm{e}}$ siècle en Angleterre, en France et en Allemagne. Cette nouvelle acception ("culture» au sens de "civilisation») est notamment détectable dans le sens que Johann Gottfried von Herder donne au terme allemand Kultur dans son ouvrage Idées pour la philosophie de l'histoire de l'humanité (1784), et dans le titre de l'ouvrage de G. F. Flemm, Allgemeine Kulturgeschichte der Menschheit (" Histoire Culturelle générale de l'Humanité »), dont les différents volumes sont publiés entre 1843 et 1852 . Ce nouveau sens s'ajoute au sens traditionnel de "culture agricole», lui-même contenu dans la racine latine du mot (le verbe colere). Ce sens métaphorique est illustré par la vision élitiste de Matthew Arnold (1869), qui définit la culture comme une poursuite de la perfection en toutes choses, afin de permettre à la société d'atteindre une harmonie sociale. Une équation commence ainsi à naître entre " culture » et " beaux-arts ». Mais il faut sans doute attribuer à Edward Burnett Tylor (1871, p.1) la première définition raisonnée du terme, dans son ouvrage Primitive Culture: "Culture or Civilisation, in its wide ethnographic sense, is that complex whole which includes knowledge, belief, art, morals, law, custom, and any other capabilities and habits acquired by man as a member of society ". Franz Boas $(1898$, p. 1) assigne quant à lui à l'anthropologie, science qu'il fonde partiellement, la mission d'explorer le développement des cultures: "It is only a few years since anthropology has begun to take its rank among other sciences, and it would seem that it is already approaching the solution of its problem - that is, laying down the laws governing the growth of culture ».

9. Notamment celle de Edward Tylor, précédemment citée, et celle de Ruth Benedict (1931, p. 806): "that complex whole which includes all the habits acquired by man as a member of society $»$.

10. Par exemple celle d'Edward Sapir (1921, p. 221) : « culture, that is,... the socially inherited assemblage of practices and beliefs that determines the texture of our lives ».

11. Le terme paraît peu adapté, il vaudrait sans doute mieux parler de définitions « symboliques » de la culture.

12. Dans un ouvrage plus récent, Bodley (1997) dresse un inventaire des définitions de la culture en se limitant au champ de l'anthropologie culturelle, et obtient une nomenclature quasiidentique à celle de Kroeber et Kluckhorn. Il dénombre en effet huit interprétations possibles : la 
culture peut être envisagée comme un ensemble de réalités observables (l'organisation sociale ou la religion par exemple); on peut la définir historiquement (elle possède un aspect patrimonial, constituant donc une tradition) ; la culture peut aussi constituer un mode de vie défini comme un ensemble de comportements sociaux «attendus »; agrégation d'idéaux et de valeurs, elle peut avoir une fonction normative ; on peut également lui donner une valeur fonctionnelle, auquel cas elle décrit la manière dont l'homme s'adapte à son environnement; elle peut servir à distinguer l'homme de l'animal, devenant alors un ensemble de comportements acquis servant à inhiber nos impulsions; certains la définissent comme une structure mettant en relation idées, comportements et symboles; et enfin, elle peut être définie comme une symbolique, c'est-à-dire un ensemble de sens attribués de manière arbitraire à certains éléments du réel sensible par une société.

13. Douglas Reeman, alias Alexander Kent, s'est engagé dans la marine royale britannique à l'âge de 16 ans. Pendant la Seconde Guerre mondiale, et la Guerre de Corée, il est officier sur plusieurs bâtiments de surface.

14. Selon Volo \& Volo (2002, p. 58), la profondeur était mesurée en brasses (fathoms) sur les bâtiments de combat de la Royal Navy au temps de la marine à voile.

15. On trouve chez Jolly (2000) un glossaire compilant les lexies constitutives de ce jargon. Par exemple, la lexie pusser's (dérivée de purser) désigne les équipements réglementaires au sein de la Royal Navy : on dira ainsi pusser's brown (le papier toilette), pusser's dust (le café en poudre servi au mess), pusser's hard (le savon réglementaire), etc.

16. Comme l'indique Sterling (2007, p. 222-223 \& p. 360-361), ce système de communication par pavillons permit de standardiser l'usage des signaux au sein de la marine britannique. Il consistait à communiquer des instructions (codifiées par un manuel officiel) au moyen d'un jeu de 21 pavillons que l'on hissait sur les mâts du bâtiment de commandement. Le bâtiment destinataire du signal devait systématiquement « envoyer l'aperçu ", c'est-à-dire hisser sur ses mâts la même combinaison de pavillons, afin de signaler la bonne réception du message. Ce système fut perfectionné en 1800 par l'amiral Home Riggs Popham, et adopté en 1803 par la Royal Navy sous l'appellation "Telegraphic Signals of Marine Vocabulary ». C'est grâce à ce système que l'amiral Nelson envoya aux bâtiments de sa flotte son fameux message «England expects that every man will do his duty » lors de la Bataille de Trafalgar.

17. Une autre discipline, la médecine de guerre au temps de la marine à voile, n'est évoquée que de manière allusive. Dans ce passage, Kent nous donne par exemple à voir son caractère très rudimentaire : "...strapped and writhing like a sacrifice on an altar was a badly wounded seaman, his leg already half amputated by Tobias Ellice, the surgeon. [...] His assistants were using all their strength to restrain the struggling victim and pin his spread-eagled body on top of the platform of sea chests, which sufficed as an operating table. The man had rolled his eyes with each nerve-searing thrust of the saw, had bitten into the leather strap between his teeth until the blood had spurted from his lips... » (TGWS, p. 108)

18. Bronner (ibid., p. 8) signale que cette cérémonie à dimension humoristique et s'apparentant à un bizutage est organisée par les marins britanniques (qui la nomment «crossing of the line »), français (le passage de la ligne), allemands (Linientaufen ou Aquatortaufen), hollandais (zeedop) et danois (hønses for linjen).

19. Par exemple The Adventures of Roderick Random, de Smollett, ou des récits tels que A New Voyage Round the World, de William Dampier, publié en 1697.

20. Ils citent à ce sujet (ibid., p. 156) l'amiral britannique Cuthbert Collingwood, qui écrit à un ami en 1808 : «I never knew a woman brought to sea in a ship that some mischief did not befall the vessel ». Cependant, il faut nuancer selon eux cette superstition misogyne, car il était fréquent que les capitaines, qui disposaient d'une grande latitude d'action, fassent embarquer leurs épouses à bord pendant toute la durée d'une campagne.

21. Nous pensons notamment aux études commanditées par l'US Army et reposant sur des enquêtes conduites auprès de très nombreux militaires au sujet de leur expérience lors du 
deuxième conflit mondial, les Studies in Social Psychology in World War II, dont les deux premiers volumes (dirigés par Stouffer, 1949) sont connus sous le titre de "The American Soldier». Ces études fondées sur le dépouillement de très nombreuses entrevues avec des G. I.'s ont permis de mettre en évidence l'espace de socialisation fondamental du militaire et notamment de l'homme du rang: le groupe primaire. Composé d'une quinzaine de personnes, le groupe primaire constitue l'environnement d'interaction sociale immédiat du membre d'équipage. Il ne recoupe pas toujours exactement les structures administratives ou fonctionnelles de l'unité militaire dans laquelle il se développe; c'est ce qu'indiquent Coates \& Pellegrin (1965, p. 312) : «In discussing primary groups in the armed forces, it is common to describe the primary group as somewhat coextensive with some small formal unit such as an infantry squad, a tank crew, an air crew, or a barracks». Le groupe primaire constitue donc une structure locale de solidarité et d'entraide, dont la survie constitue, à en croire la sociologie militaire, le principal facteur de motivation des hommes au combat.

22. Terme signifiant « navire de guerre ».

23. Voir dans notre bibliographie Vercken, 1986.

\section{RÉSUMÉS}

Mettant en scène Richard Bolitho, officier de la marine britannique au temps de la Révolution française et des guerres napoléoniennes, les 29 romans d'aventures maritimes publiés par Alexander Kent (alias Douglas Reeman, ex-officier de la Royal Navy) offrent un terrain d'investigation idéal pour qui souhaite s'interroger sur le substrat culturel spécialisé qui se manifeste dans certaines fictions. Le matériau culturel y est en effet dense, quoique hétérogène : éléments langagiers (le jackspeak, jargon des marins britanniques), mais aussi traditions observées dans la vie à bord des bâtiments, situations et gestes empreints d'une portée symbolique, règles implicites encadrant les actions militaires (le jus in bello). En prenant appui sur trois ouvrages (Cap sur la gloire, En ligne de bataille, et Ennemi en vue) appartenant à ce cycle romanesque qui s'inscrit dans la tradition littéraire du sea yarn (le roman d'aventures maritimes), il conviendra dans un premier temps de s'interroger sur la légitimité de la FASP en tant que nouvelle voie d'accès aux cultures des milieux spécialisés. Celles-ci peuvent être caractérisées par certains invariants: historicité, territorialité, viscosité, aspect patrimonial. Les cultures spécialisées paraissent constituer un champ d'investigation primordial dès lors qu'on s'intéresse à la spécialisation de la langue, par exemple à ce qu'on pourrait appeler ici l'« anglais maritime ». En effet, le primum mobile de cette spécialisation langagière semble être d'essence culturelle, ce qui nous invite à une démarche holistique dès lors qu'on souhaite contribuer à la caractérisation de certains discours spécialisés.

The cultural traits peculiar to a professional milieu can be explored through ethnographic field work, but also through a certain strain of fiction in which what Petit (1999) dubbed the "professional substratum" seems to be at the forefront. Such "substratum-rich novels" (SRNs) provide a vantage point from which to explore the culture of a given milieu. In this paper, we investigate the cultural substratum underlying Alexander Kent's franchise of 29 maritime novels. Spanning the periods of the French Revolution and of the Napoleonic wars, and following the life of Richard Bolitho, a fictitious naval officer, these novels provide a vivid insight into life on board Royal Navy warships in the age of sail. Acknowledging the fact that the term "culture" remains 
difficult to define, we sought to identify culture-related elements in three of Kent's "sea yarns" ( Enemy In Sight!, To Glory We Steer, and Form Line of Battle!). It appears that Kent's rendering of maritime culture is based on a combination of heterogeneous elements such as naval traditions, symbolic gestures and ceremonies, customary rules (e.g. jus in bello on implicit guidelines for fighting in a "moral" way), and even "jackspeak" (the British Tar's specific parlance). A former naval officer himself, Alexander Kent obviously drew on his own professional experience to provide the reader with a relatively reliable "cultural portrait" of the Royal Navy in the age of sail. Kent's SRNs can thus be viewed as an ideal hunting ground for researchers interested in maritime English and maritime culture at large. More broadly, it appears that analyzing the specialized cultural substratum embedded in SRNs may be useful to gain better understanding of the interactions between specialized cultures and specialized discourses.

INDEX

Mots-clés : FASP, anglais maritime, culture professionnelle

Keywords : Naval fiction, maritime English, professional culture

\section{AUTEUR}

\section{ANTHONY SABER}

Maître de conférences à l'ENS de Cachan, Équipe ASPDA/EA 4140 